\title{
Evaluation of the uptake function of liver in rats with obstructive jaundice before and after relief from obstruction by superparamagnetic iron oxide-enhanced magnetic resonance imaging
}

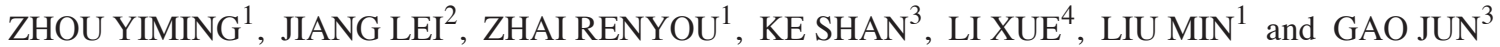 \\ ${ }^{1}$ Department of Radiology, Beijing Chao-Yang Hospital, Capital Medical University, Chaoyang District, Beijing 100020; \\ ${ }^{2}$ Department of Radiology, Beijing Hospital, Dongcheng District, Beijing 100730; ${ }^{3}$ Department of Hepatobiliary Surgery, \\ Beijing Chao-Yang Hospital (Jingxi Campus), Capital Medical University, Shijingshan District, Beijing 100043; \\ ${ }^{4}$ Department of Pathology, Beijing Chao-Yang Hospital, Capital Medical University, \\ Chaoyang District, Beijing 100020, P.R. China
}

Received January 18, 2012; Accepted May 14, 2012

DOI: $10.3892 / \mathrm{ol} .2012 .729$

\begin{abstract}
The aim of this study was to investigate the changes in the uptake function of the liver in rats with obstructive jaundice prior to and following relief from obstructive jaundice, and to investigate whether superparamagnetic iron oxide (SPIO)-enhanced magnetic resonance imaging (MRI) could be used to assess liver uptake function. In total, 40 male Sprague Dawley rats were randomly assigned into four groups: Obstructive jaundice, internal drainage, external drainage and sham surgery. The common bile ducts in the obstructive jaundice group were ligated. Internal drainage (ID) and external drainage (ED) groups were ligated, followed by internal drainage or external drainage, respectively. The T2 and T2* values of the liver parenchyma were measured. Liver sections were stained with Perls' Prussian blue, and hematoxylin and eosin. The number of SPIO-nanoparticle clusters was counted manually using a microscope. Total bilirubin of the blood was measured. Results showed that the T2 and T2* values and total bilirubin of the obstructive jaundice group were significantly higher compared to the other three groups. The number of SPIO-nanoparticle clusters in the obstructive jaundice group was significantly lower compared to the other three groups. In conclusion, obstructive jaundice suppresses liver uptake function in rats, which may be reversed by internal and external
\end{abstract}

Correspondence to: Dr Zhai Renyou, Department of Radiology, Beijing Chao-Yang Hospital, Capital Medical University. No 8th, Gongren Tiyuchang Nanlu, Chaoyang District, Beijing 100020, P.R. China

E-mail: ryzhai219@hotmail.com

Key words: liver uptake function, internal and external drainage, obstructive jaundice, superparamagnetic iron oxide-enhanced magnetic resonance imaging biliary drainage. However, no significant difference was found between the therapeutic effect of ID and ED on liver uptake function. Thus, SPIO-enhanced MRI may be used to evaluate the uptake function of the liver.

\section{Introduction}

Patients with obstructive jaundice (OJ) are at high risk of postoperative complications $(1,2)$, such as endotoxemia, renal failure, and even mortality. OJ is associated with dysfunction of liver uptake function (3). Several previous studies have shown that the liver uptake function was severely suppressed in patients and rats with biliary obstruction (4-6). Usually OJ is depressed by internal drainage (ID) or external drainage (ED), but it is indeterminate whether there is any difference between the effects of ID and ED on liver uptake function.

Superparamagnetic iron oxide (SPIO) is a liver-specific magnetic resonance imaging (MRI) contrast agent. The technique relies on the ability of the liver to take up SPIO particles $(7,8)$. The current clinical application of SPIO is mainly to detect hepatocellular carcinoma (9-11), and few studies are available on the application of SPIO in OJ (12).

This study investigated whether the SPIO-enhanced MRI could be effectively used to evaluate the liver uptake function in OJ rats, and consequently revealed the effect of ID and ED on liver uptake function.

\section{Materials and methods}

Animals. In total, 40 male Sprague Dawley rats weighing 300-400 g were used in this study. The animals were housed in a specific-pathogen-free environment in the Laboratory Animal Service Center of Capital Medical University, and fed with standard rat chow. The animals were randomly divided into four groups: Sham surgery $(\mathrm{SH}, \mathrm{n}=10)$, OJ $(\mathrm{n}=10)$, ID $(n=10), E D(n=10)$. The laparotomy in all groups was made through a $4-5 \mathrm{~cm}$ upper middle abdominal incision. The 
following protocol was applied: i) SH group: Common bile duct was only divided. ii) OJ group: The common bile duct was ligated twice with 5/0 silk. iii) ED group: A 19G (approximately $1 \mathrm{~mm}$ in diameter) epidural catheter was inserted into the common bile duct, and the other end of the catheter exteriorized at the nape of the neck through the subcutaneous channel. The outlet of the catheter was ligated for 14 days and then drained for 14 days. iv) ID group: One catheter was inserted into the common bile duct. Another catheter was inserted into the jejunum, and the outer end of the catheter was also exteriorized at the nape of the neck. The outlets of the common bile duct catheter and the jejunum catheter were connected by an elastic tube. The tube was ligated for 14 days. On the 15th day, the ligation was removed and drainage lasted for 14 days. Two of the 40 animals died during the study. One was in the ID group, and the other was in the ED group.

The SH and OJ group were scanned by SPIO-MRI, and sacrificed for liver and blood collection 14 days following surgery. The same procedure was applied in the ID and ED groups after 14 days of drainage. The study was approved by the Ethics Committee of Beijing Chaoyang Hospital

SPIO contrast agent. Resovist (Schering Ag, Germany) was used as the SPIO contrast agent, and is a hydrophilic colloidal solution of SPIO coated with carboxydextran, which forms ferucarbotran. The diameter of the nanoparticle is $62 \mathrm{~nm}$. The contrast agent was administered via a tail vein or femoral vein (30 $\mu \mathrm{mol} \mathrm{Fe} / \mathrm{kg})$.

MRI scan. Anesthesia was induced with an intraperitoneal injection of $10 \%$ chloral hydrate $(3.5 \mathrm{ml} / \mathrm{kg}$ ) (made by Beijing Chaoyang Hospital). The rats were placed in a plastic container to restrict the respiratory movement. The MRI was performed with a 1.5-T superconducting imaging system (Signa HDx 1.5T; GE Healthcare, Waukesha, WI, USA) $1 \mathrm{~h}$ following the administration of SPIO. Scout images were obtained first. The $\mathrm{T} 2$ value was acquired by the $\mathrm{T} 2$ mapping sequence with 15 readout echoes: $\mathrm{TR}=1025 \mathrm{~ms}$, first TE $/ \Delta \mathrm{TE}=7.8 / 7.8 \mathrm{~ms}$, flip angle $=30^{\circ}, \mathrm{FOV}=80 \times 80 \mathrm{~mm}$, scan matrix $=160 \times 128$, slice thickness $=3.0 \mathrm{~mm}$, bandwidth $=31.25 \mathrm{~Hz}, \mathrm{NEX}=4$. The scan time was $8 \mathrm{~min} 44 \mathrm{sec}$. The T2* value was obtained by the $\mathrm{T} 2 *$ mapping sequence with 15 readout echoes: $\mathrm{TR}=150 \mathrm{~ms}$, first TE $/ \Delta \mathrm{TE}=2.6 / 4.4 \mathrm{~ms}$, flip angle $=20^{\circ}, \mathrm{FOV}=80 \times 80 \mathrm{~mm}$, scan matrix $=128 \times 128$, slice thickness $=3 \mathrm{~mm}$, bandwidth $=$ $31.25 \mathrm{~Hz}, \mathrm{NEX}=2$. The scan time was $2 \mathrm{~min} 38 \mathrm{sec}$.

Analysis of MRI data. The T2 and T2* value analysis was performed with MRI workstation packaged software: functool-T2MAP. The region of interest (ROI) boxes were oval and placed in both the right and left lobes of the liver. The ROIs were selected to be as large as possible, with exclusion of any focal hepatic lesions and major branches of the portal and hepatic veins. The ROI boxes were placed on three slices through the center of the liver, and the mean $\mathrm{T} 2$ and $\mathrm{T} 2 *$ values of the right and left lobe were calculated, respectively.

Kupffer cells in liver sections. Each liver was fixed with $10 \%$ formalin. Liver sections were stained with hematoxylin and eosin (H\&E) and Perl's Prussian blue. Hepatic lobule structure and SPIO nanoparticles were observed and imaged under a microscope (Olympus microscope, BX41). The number of SPIO-nanoparticle clusters was counted manually in three high-power fields (x200), and the mean was calculated.

Statistical analysis. Data were shown as the means \pm standard deviation (SD). Statistical analysis was performed using SPSS version 17.0 for Windows (Chicago, IL, USA). One-way ANOVA followed by Tukey's honestly significant difference (HSD) test, Kruskal-Wallis test and Mann-Whitney U test were used to evaluate statistical significance. $\mathrm{P}<0.05$ was considered to indicate a statistically significant difference.

\section{Results}

$T 2$ and $T 2 *$ values. In order to determine the uptake function of the liver, T2 and T2* values were evaluated (Fig. 1). These values are quantitative parameters for liver parenchyma, which can be reduced by SPIO nanoparticles. The T2 values in the OJ group are significantly higher $(44.3 \pm 2.2)$ than in the other groups. There was a significant decrease of the $\mathrm{T} 2$ values in the SH $(25.7 \pm 2.4)$, ID $(27.9 \pm 3.3)$ and $\mathrm{ED}(28.5 \pm 2.7)$ groups $(\mathrm{P}<0.001)$. In a similar manner the $\mathrm{T} 2 *$ value in the SH (0.6 \pm 0.4$),$ ID $(0.4 \pm 0.3)$ and ED $(0.8 \pm 0.5)$ groups decreased significantly, whereas the $\mathrm{T} 2 *$ value in the OJ group was significantly higher $(12.6 \pm 1.8 ; \mathrm{P}<0.001)$ (Fig. 2).

Liver section and SPIO nanoparticle clusters. Photomicrographs of liver tissue stained by H\&E revealed the histological structure of the liver. The OJ group showed the typical obstructive jaundice histological changes: proliferation of bile ductules, neutrophil infiltration in sinusoid, hepatic lobule reconstruction and intracellular bile retention. The histological changes in the ID and ED groups were much milder than in the OJ group (Fig. 3).

The liver section stained by Perls' Prussian blue revealed the number of SPIO nanoparticle clusters (Fig. 4). The number of SPIO nanoparticle clusters in the OJ group $(4.2 \pm 1.3)$ was significantly lower than in the other three groups $(\mathrm{P}<0.001)$ (Fig. 5). No SPIO-nanoparticles were identified in certain OJ group sections.

Total bilirubin. Total bilirubin was used to assess the severity of OJ (Fig. 6). The total bilirubin in OJ $(149.1 \pm 36.7 \mu \mathrm{mol} / \mathrm{l})$ rats was significantly higher $(\mathrm{P}<0.001)$ than in the other groups. No significant difference was found among the $\mathrm{SH}$ (6.4 $\pm 2.3 \mu \mathrm{mol} / \mathrm{l}), \mathrm{ID}(9.4 \pm 2.3 \mu \mathrm{mol} / \mathrm{l})$ and $\mathrm{ED}(18.6 \pm 8.8 \mu \mathrm{mol} / \mathrm{l})$ groups.

\section{Discussion}

OJ patients have an increased risk of complications and mortality. Liver uptake dysfunction is important in these processes. It is responsible for the clearance of bacteria and lipopolysaccharides from the intestines. Several studies in the past have shown that the uptake function of the liver was severely suppressed in patients and rats with biliary obstruction (4-6). In the above-mentioned studies, the vascular clearance method was used to evaluate liver uptake function. A series of different test particles, including, synthetic dyes, colloidal carbon and radiolabelled bacteria have been 


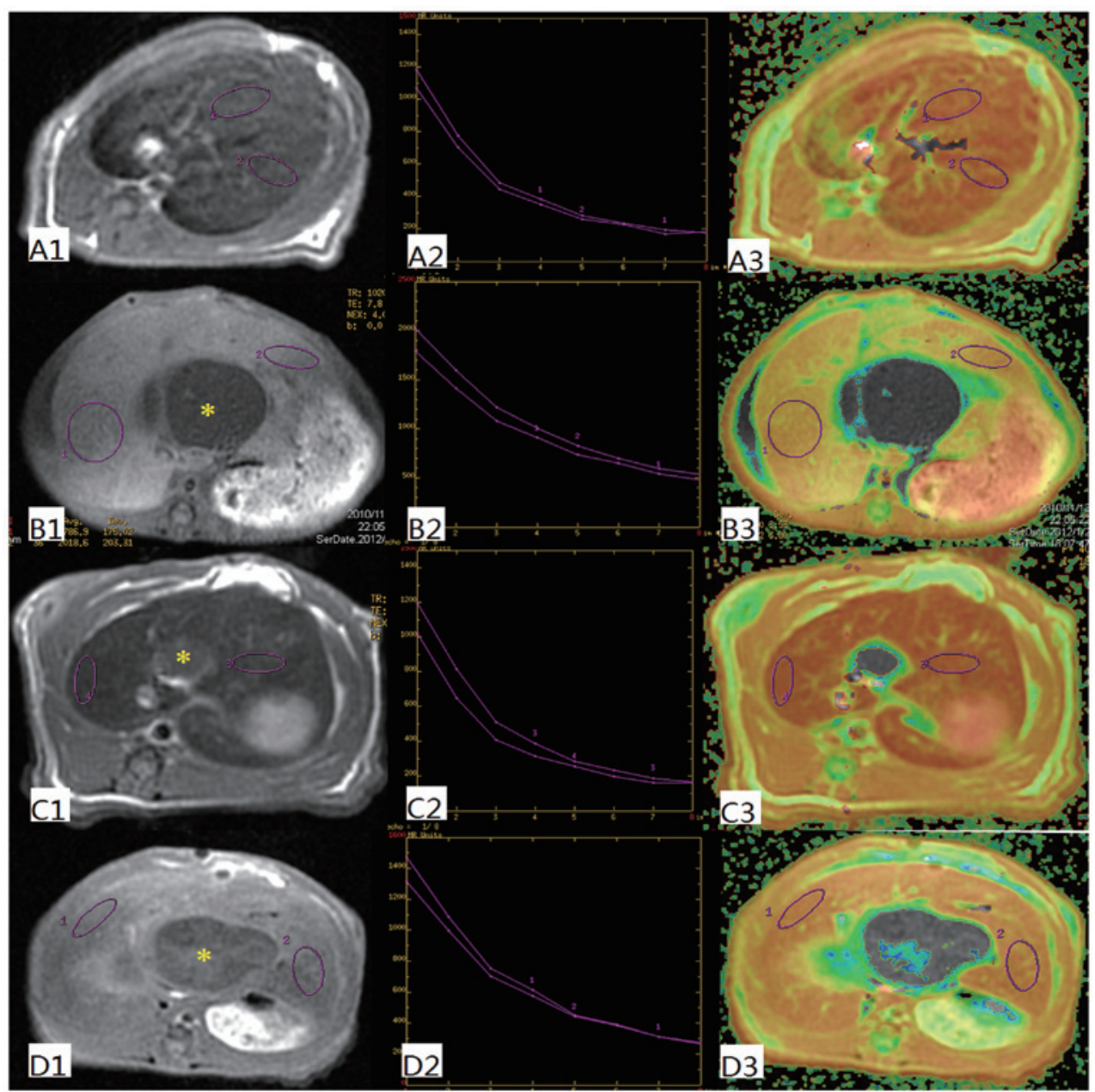

Figure $1 . \mathrm{T} 2$ and $\mathrm{T} 2 *$ values reflect $\mathrm{T} 2$ transverse relaxation time and $\mathrm{T} 2 *$ relaxation time, respectively. A multiecho technique was used to measure $\mathrm{T} 2$ and T2* values. 1) Original data images. 2) The signal intensity of each region of interest was fitted to one exponential. 3) A color map represented the variations in relaxation time. (A) OJ group; (B) SH group; (C) ID group; (D) ED group. OJ, obstructive jaundice; SH, sham surgery; ID, internal drainage; ED, external drainage.

used. However, these methods are invasive or radiologically harmful, and cannot be applied clinically. Therefore, this study was designed to use SPIO-enhanced MRI to assess the liver uptake function.

SPIO is a liver-specific MRI contrast agent. The agent is capable of shortening the $\mathrm{T} 2$ transverse relaxation time and $\mathrm{T} 2 *$ relaxation time of liver, so that the $\mathrm{T} 2$ and $\mathrm{T} 2 *$ values decrease. Previously, SPIO was mainly used to differentiate hepatocellular carcinoma from benign focal lesions. Recently, some investigators have assessed the application of SPIO in certain diffuse diseases, such as non-alcoholic fatty liver disease, liver cirrhosis or fibrosis (13-15). These studies showed that following the administration of SPIO, the signal in the model group exhibited a significantly lower reduction compared to the normal group. The signal in the severe disease group showed a lower reduction compared to the mild group. This phenomenon was repeated in animal models and patients. These studies indicated that diffuse liver diseases impair liver uptake function, which may be identified by SPIO-enhanced MRI.

$\mathrm{T} 2$ and $\mathrm{T} 2 *$ values of the liver in the OJ group are significantly higher than in the other groups. This suggested that there were less SPIO-nanoparticles in the liver parenchyma

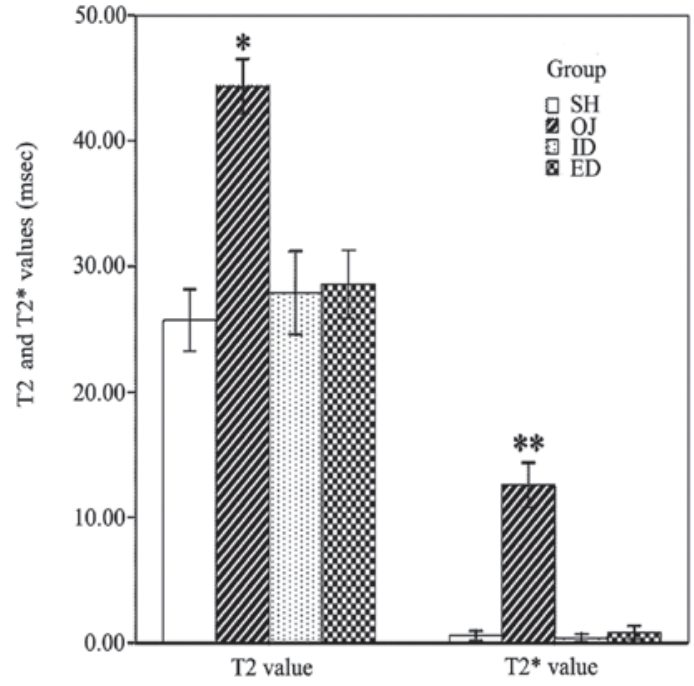

Figure 2 . The T2 and T2* values were decreased by SPIO nanoparticles. The higher T2 and T2* values suggested fewer SPIO-nanoparticles in the liver parenchyma. The T2 values in the OJ group are significantly higher $\left({ }^{*} \mathrm{P}<0.001\right.$, by one-way ANOVA and Tukey's HSD test) than in the other groups. Similarly, the $\mathrm{T} 2 *$ value in the OJ group was significantly higher $\left(12.6 \pm 1.8 ;{ }^{* *} \mathrm{P}<0.001\right.$, by Kruskal-Wallis test and Mann-Whitney U test) than the other groups. SPIO, superparamagnetic iron oxide; OJ, obstructive jaundice; HSD, honestly significant difference. 


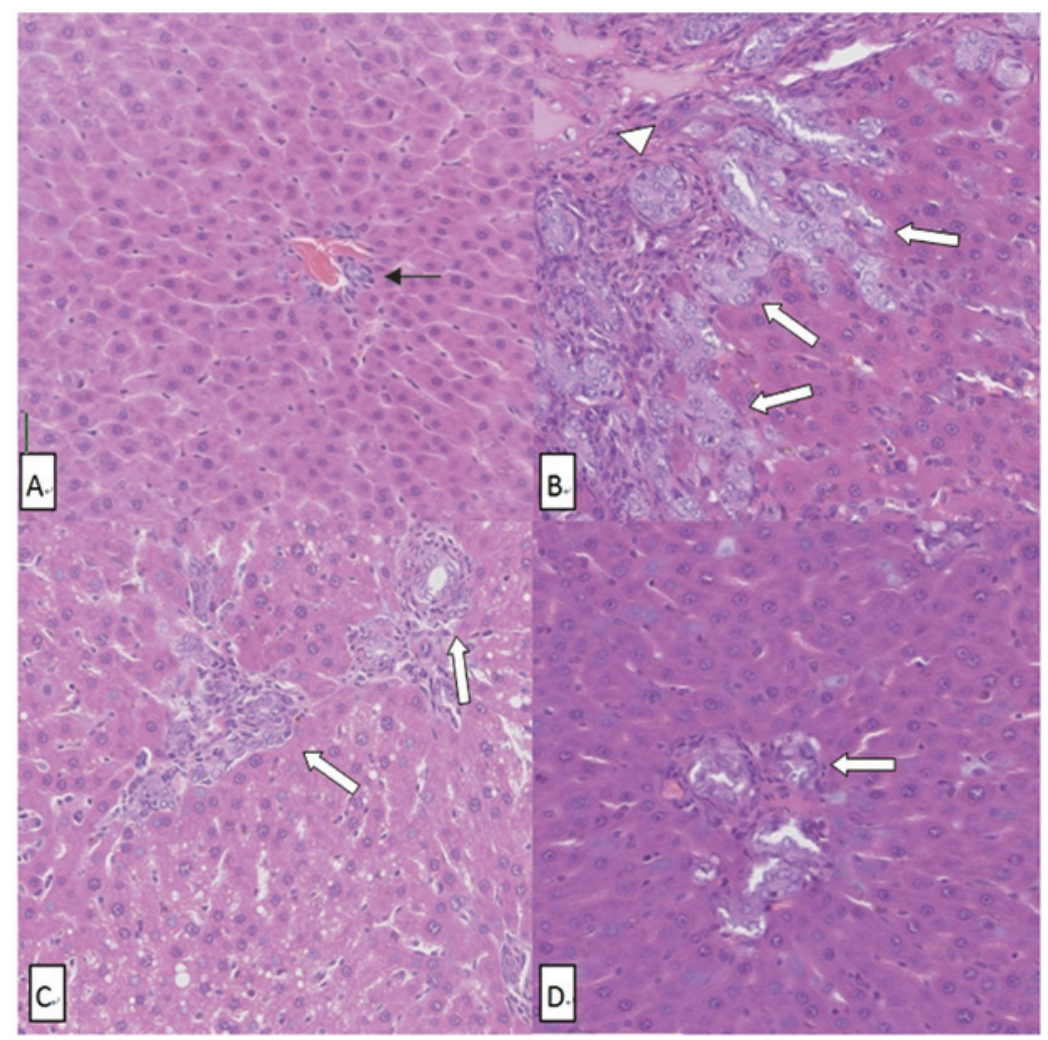

Figure 3. Photomicrographs of H\&E-stained tissue slices (x200). (A) SH group. Hepatic lobules and bile ductules were intact. Black arrow, normal bile ductules. (B) OJ group. Photomicrographs showed the typical obstructive jaundice histological change. White arrow, proliferation of bile ductules; white arrow head, neutrophils are hardly observed in normal situations, indicating an inflammation reaction in the liver. Hepatic lobules cannot be identified. (C) ID group. (D) ED group: The histological changes were much milder than the OJ group. Proliferation of bile ductules was much milder (white arrow). Some hepatic lobules were evident; neutrophils were rarely observed. H\&E, hematoxylin eosin; OJ, obstructive jaundice; SH, sham surgery; ID, internal drainage; ED, external drainage.

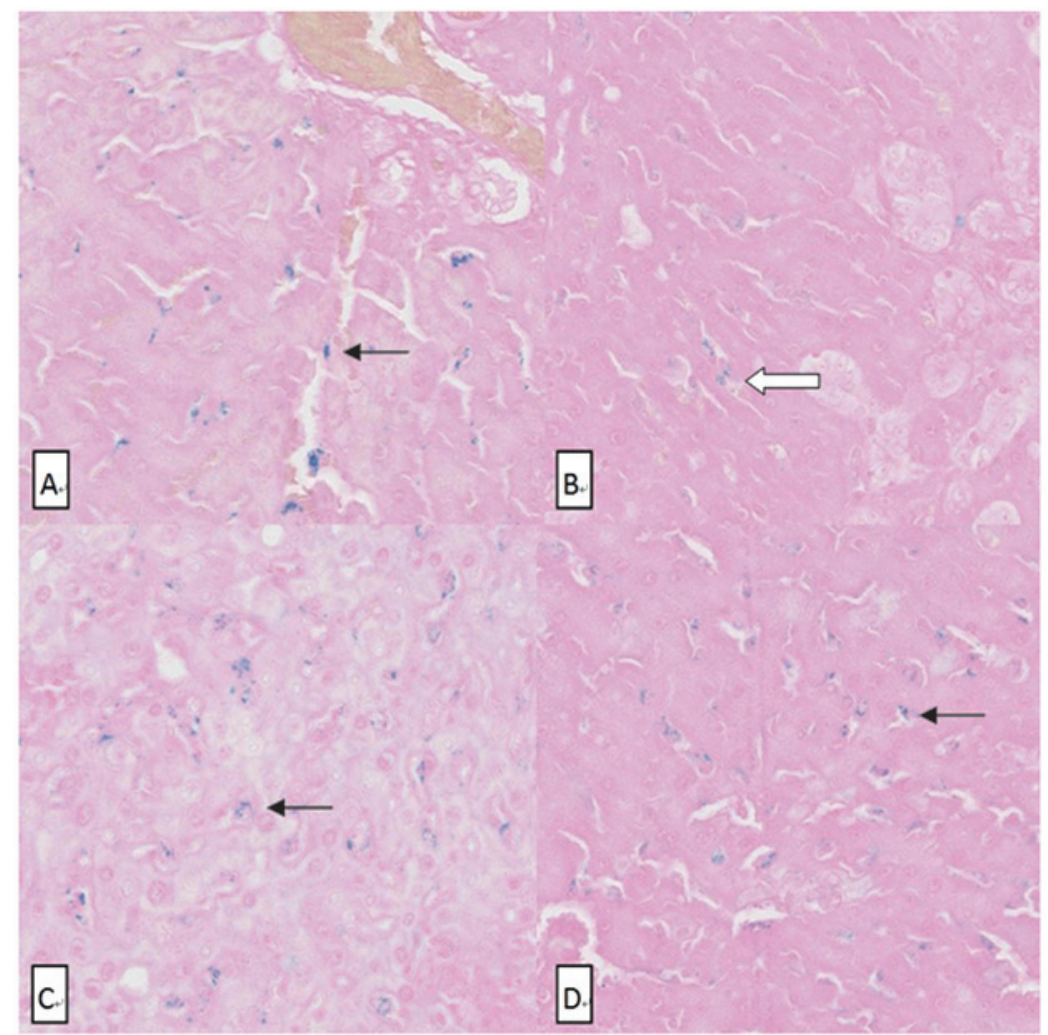

Figure 4. Photomicrographs of Perls' Prussian blue-stained liver slices (x200). Blue dots are the SPIO nanoparticles. (A) SH group; (B) OJ group; (C) ID group; (D) ED group. Black arrow: The blue spots were numerous and dense in the SH, ID and ED groups; white arrow: The blue spots were rare and light in the OJ group. SPIO, superparamagnetic iron oxide; OJ, obstructive jaundice; SH, sham surgery; ID, internal drainage; ED, external drainage. 


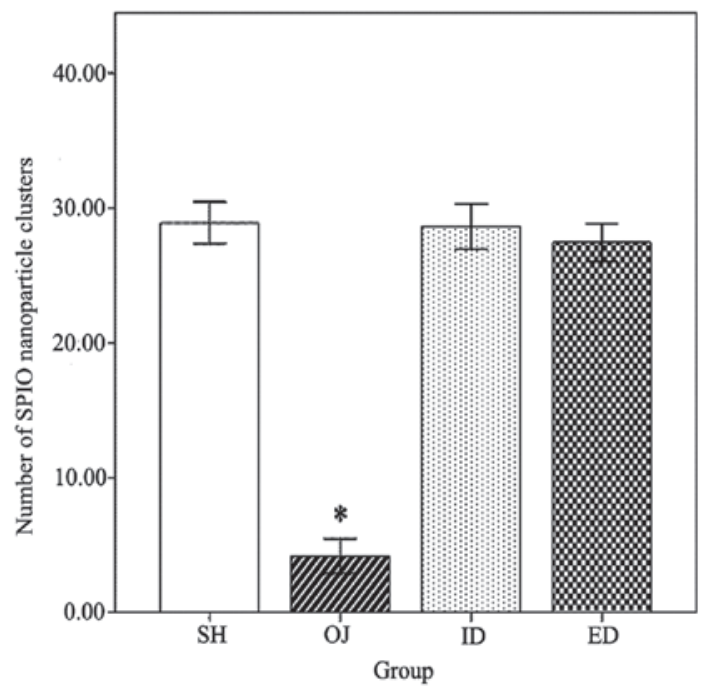

Figure 5. The number of SPIO nanoparticle clusters in liver sections reflects the liver uptake function. SPIO nanoparticle clusters in the OJ group were significantly lower than in the other three groups ( $\mathrm{P}<0.001$, by one-way ANOVA and Tukey's HSD test). SPIO, superparamagnetic iron oxide; OJ, obstructive jaundice; HSD, honestly significant difference; $\mathrm{SH}$, sham surgery; ID, internal drainage; ED, external drainage.

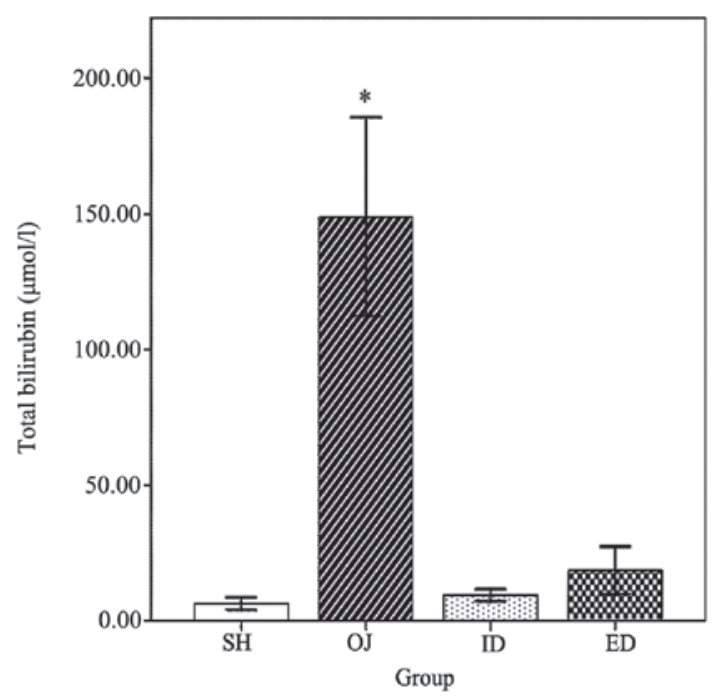

Figure 6. Total bilirubin was used to the assess the severity of OJ. The total bilirubin in OJ rats was significantly higher than that in the other groups ( $\mathrm{P}<0.001$, by Kruskal-Wallis test and Mann-Whitney U test). OJ, obstructive jaundice; $\mathrm{SH}$, sham surgery; ID, internal drainage; ED, external drainage.

due to the depression of liver uptake function $(16,17)$. There were much fewer SPIO-nanoparticles observed in the OJ group liver sections than in the other three groups. Additionally, no SPIO-nanoparticle clusters were observed in many areas of the OJ group section. There was no significant difference between the ID group and $\mathrm{ED}$ group when the $\mathrm{T} 2$ value, $\mathrm{T} 2 *$ value and the number of SPIO-nanoparticle clusters were compared. No significant differences were found between the therapeutic effect of ID and ED on liver uptake function.

In Perls' Prussian blue staining of liver sections, SPIOnanoparticles taken up by liver are shown as blue dots. Thus, the number of blue dots in liver sections reflects the liver uptake function. The number of blue dots was counted and normalized for each sample, which was used as the standard method to assess the uptake function of the liver in our study.

The total bilirubin and histological changes of liver sections showed that the OJ model and the drainage model were successful. The total bilirubin of the OJ group was significantly higher than that of the SH group. No significant difference was observed among the total bilirubin of the ID, $\mathrm{ED}$ and $\mathrm{SH}$ groups. The photomicrographs of the H\&E-stained tissue slices showed typical obstructive jaundice histological characteristics. In the ID and ED groups, the histological findings were much milder. The obstructive jaundice historical changes in the ID and ED groups partially reversed, indicating histological reverse following functional recovery.

Previous studies have proven that biliary decompression improved liver uptake function (4-6). In the present study, SPIO-enhanced MRI also confirmed this phenomenon. No significant difference was found between the ID and ED groups when the $\mathrm{T} 2$ and $\mathrm{T} 2 *$ values were compared. Clements et al reported that rats undergoing internal biliary drainage had a significantly increased liver uptake function compared with the rats treated by ED (18). We attributed the difference between the two studies to the different drainage models used. In our study, rats in the ID and ED groups underwent a single surgical procedure. The biliary obstruction and decompression were obtained by obstructing and releasing the catheter placed outside the rats. This procedure minimized the effect of surgery on rats. In Clements et al's study, the biliary obstruction and decompression were obtained by laparotomy performed twice. There were two control groups in their study: sham/ED and sham/ID. The rats of these two groups underwent ED or ID procedures, respectively, without OJ. Their results showed that the liver uptake function of the sham/ED group was lower than the sham/ID group. Therefore, we assumed that their ED surgery may depress the liver uptake function.

In this study, we found that: i) SPIO-enhanced MRI could be used to assess the liver uptake function in certain liver conditions, such as OJ, and patients underwent biliary relief; ii) OJ suppresses liver's uptake function in rats. Internal and external biliary drainage is capable of reversing the change in the uptake function of Kupffer cells. Our study did not show a significant difference between the therapeutic effect of ID and $\mathrm{ED}$ on liver uptake function. This suggests that ED may be enough for OJ patients clinically.

\section{Acknowledgements}

This study was supported by the Chinese National Key Technology R\&D Program No. 2007BAI05B06, and the Chinese National Scientific Research Foundation No. 30900364.

\section{References}

1. Tomioka M, Iinuma $\mathrm{H}$ and Okinaga $\mathrm{K}$ : Impaired Kupffer cell function and effect of immunotherapy in obstructive jaundice. J Surg Res 92: 276-282, 2000.

2. Ljungdahl M, Osterberg J, Ransjo U, Engstrand L and Haglund U: Inflammatory response in patients with malignant obstructive jaundice. Scand J Gastroenterol 42: 94-102, 2007.

3. Scott-Conner CE and Grogan JB: The pathophysiology of biliary obstruction and its effect on phagocytic and immune function. J Surg Res 57: 316-336, 1994. 
4. Charlier N, Neyrinck AM, Beghein N, Delzenne NM and Gallez B Assessment of liver phagocytic activity using EPR spectrometry and imaging. Magn Reson Imaging 27: 565-569, 2009.

5. Ding JW, Andersson R, Stenram U, Lunderquist A and Bengmark S: Effect of biliary decompression on reticuloendothelial function in jaundiced rats. Br J Surg 79: 648-652, 1992.

6. Megison SM, Dunn CW, Horton JW and Chao H: Effects of relief of biliary obstruction on mononuclear phagocyte system function and cell mediated immunity. Br J Surg 78: 568-571, 1991.

7. Nishie A, Yoshimitsu K, Nakayama T, et al: In vitro imaging of human monocytic cellular activity using superparamagnetic iron oxide. Comput Med Imaging Graph 31: 638-642, 2007.

8. Briley-Saebo K, Bjornerud A, Grant D, Ahlstrom H, Berg T and Kindberg GM: Hepatic cellular distribution and degradation of iron oxide nanoparticles following single intravenous injection in rats: implications for magnetic resonance imaging. Cell Tissue Res 316: 315-323, 2004.

9. Kim T, Murakami T, Hori M, Onishi H, Tomoda K and Nakamura H: Effect of superparamagnetic iron oxide on tumorto-liver contrast at T2*-weighted gradient-echo MRI: comparison between 3.0T and 1.5T MR systems. J Magn Reson Imaging 29: 595-600, 2009

10. Namkung S, Zech CJ, Helmberger T, Reiser MF and Schoenberg SO: Superparamagnetic iron oxide (SPIO)-enhanced liver MRI with ferucarbotran: efficacy for characterization of focal liver lesions. J Magn Reson Imaging 25: 755-765, 2007.

11. Tanimoto A and Kuribayashi S: Application of superparamagnetic iron oxide to imaging of hepatocellular carcinoma. Review. Eur J Radiol 58: 200-216, 2006.
12. Saito $\mathrm{T}$, Abe $\mathrm{T}$, Tsuchiya $\mathrm{T}$, et al: Sequential magnetic resonance imaging for evaluation of Kupffer cell function. Hepatogastroenterology 55: 596-599, 2008.

13. Lucidarme O, Baleston F, Cadi M, et al: Non-invasive detection of liver fibrosis: Is superparamagnetic iron oxide particleenhanced MR imaging a contributive technique. Eur Radiol 13: 467-474, 2003.

14. Tomita K, Tanimoto A, Irie R, et al: Evaluating the severity of nonalcoholic steatohepatitis with superparamagnetic iron oxide-enhanced magnetic resonance imaging. J Magn Reson Imaging 28: 1444-1450, 2008.

15. Tanabe M, Ito K, Shimizu A, et al: Hepatocellular lesions with increased iron uptake on superparamagnetic iron oxideenhanced magnetic resonance imaging in cirrhosis or chronic hepatitis: comparison of four magnetic resonance sequences for lesion conspicuity. Magn Reson Imaging 27: 801-806, 2009.

16. Kalber TL, Smith CJ, Howe FA, et al: A longitudinal study of $\mathrm{R} 2 *$ and $\mathrm{R} 2$ magnetic resonance imaging relaxation rate measurements in murine liver after a single administration of 3 different iron oxide-based contrast agents. Invest Radiol 40: 784-791, 2005.

17. Liu W, Dahnke H, Rahmer J, Jordan EK and Frank JA: Ultrashort $\mathrm{T} 2 *$ relaxometry for quantitation of highly concentrated superparamagnetic iron oxide (SPIO) nanoparticle labeled cells. Magn Reson Med 61: 761-766, 2009.

18. Clements WD, McCaigue M, Erwin P, Halliday I and Rowlands BJ: Biliary decompression promotes Kupffer cell recovery in obstructive jaundice. Gut 38: 925-931, 1996. 